OPEN ACCESS

Edited by:

David A. Gewirtz,

Virginia Commonwealth University,

United States

Reviewed by:

Loredana Bergandi,

University of Turin, Italy

Tareq Saleh,

Hashemite University, Jordan

*Correspondence: Xinsheng Yao

tyaoxs@jnu.edu.cn

Youwei Zhang

yxz169@case.edu

Jinshan Tang

gztangjinshan@126.com

Specialty section:

This article was submitted to Pharmacology of Anti-Cancer Drugs,

a section of the journal

Frontiers in Pharmacology

Received: 05 April 2020

Accepted: 26 May 2020

Published: 09 June 2020

Citation:

Wang F, Mayca Pozo F, Tian D, Geng $X$, Yao X, Zhang $Y$ and Tang J (2020) Shikonin Inhibits Cancer

Through P21 Upregulation and Apoptosis Induction.

Front. Pharmacol. 11:861. doi: 10.3389/fphar.2020.00861

\section{Shikonin Inhibits Cancer Through P21 Upregulation and Apoptosis Induction}

\author{
Fangfang Wang ${ }^{1,2}$, Franklin Mayca Pozo ${ }^{3}$, Danmei Tian ${ }^{1,2}$, Xinran Geng ${ }^{3}$, \\ Xinsheng Yao ${ }^{1,2 *}$, Youwei Zhang ${ }^{3 *}$ and Jinshan Tang ${ }^{1,2 *}$ \\ 1 Institute of Traditional Chinese Medicine and Natural Products, Guangdong Province Key Laboratory of Pharmacodynamic \\ Constituents of Traditional Chinese Medicine and New Drug Research, College of Pharmacy, Jinan University, Guangzhou, \\ China, ${ }^{2}$ International Cooperative Laboratory of Traditional Chinese Medicine Modernization and Innovative Drug \\ Development of Chinese Ministry of Education (MOE), College of Pharmacy, Jinan University, Guangzhou, China, \\ ${ }^{3}$ Department of Pharmacology, Case Comprehensive Cancer Center, Case Western Reserve University School of Medicine, \\ Cleveland, $\mathrm{OH}$, United States
}

Shikonin is a natural naphthoquinone compound and has demonstrated potent anticancer activities; however, the underlying molecular mechanisms remained elusive. Here we report that Shikonin inhibited the growth of a wide range of human cancer cell lines, illustrating a broad anticancer effect. Mechanistically, we show that Shikonin arrested the cell cycle at the G2/M phase, inhibited the ERK-dependent cell growth signal, and induced cell death in both P53 wild type and mutant cancer cells, which collectively contributed to the growth inhibitory effect of Shikonin. A pan-apoptosis inhibitor largely suppressed Shikonin-induced cell death, suggesting an important role of apoptosis in this process. Intriguingly, Shikonin also activated autophagy and inhibition of autophagy by depleting critical autophagic genes further increased Shikonin-induced cell death, indicating a protective role of autophagy. In uncovering the molecular mechanisms underlying these effects of Shikonin, we found that Shikonin induced a robust upregulation of P21 independent of the P53 status, upregulated autophagy genes, as well as inhibited expression of genes required for cell growth. Using mouse tumor models, we confirmed the strong anticancer effect of Shikonin in vivo. Together, our data reveal a broad range of pharmacological functions of Shikonin, involving simultaneous growth inhibition, cell cycle arrest, autophagy activation and apoptosis induction through regulating expression of critical genes involved in these pathways. Our study may facilitate the development of Shikonin in cancer therapy as a single agent or in combination with other anticancer therapies.

Keywords: Shikonin, anticancer effect, cell cycle arrest, autophagy, apoptosis, P21

\section{INTRODUCTION}

Zicao (purple gromwell), the dried root of Lithospermum erythrorhizon Sieb et Zucc, Arnebia euchroma (Royle) Johnst, or Arnebia guttata Bunge, is an herbal medicine that has been used to treat many kinds of illnesses in China and other Asian and European countries for centuries. A large number of studies have reported a wide range of biological activities of Zicao extracts including anti- 
inflammation, anti-oxidative stress, anti-virus, anti-bacteria and anti-cancer in both cultured cells and in animal models (Papageorgiou et al., 1999; Chen et al., 2002; Andujar et al., 2013; Wang et al., 2019). Shikonin is a major component of Zicao and belongs to the naphthoquinone family compound. Consistent with the reported function of Zicao extracts, Shikonin has demonstrated a broad spectrum of bioactivities including wound healing (Mani et al., 2004), anti-inflammation (Tanaka et al., 1986), anti-HIV (Chen et al., 2003), anti-cancer (Sankawa et al., 1977), and so on. It appears that its toxicity to normal tissues and organs is limited; hence, Shikonin has been extensively studied as an anti-cancer agent and had demonstrated promising effects both in vitro and in vivo (Papageorgiou et al., 1999; Chen et al., 2002; Andujar et al., 2013; Wang et al., 2019).

The molecular mechanisms underlying the anti-cancer activity of Shikonin seemed to be complicated and may depend on the cellular context (Wang et al., 2019). So far, the reported cellular targets of Shikonin include the pyruvate kinase isoenzyme M2 (PKM2) (Chen et al., 2011; Lu et al., 2018; Tang et al., 2018b), the MAPK pathway (Mao et al., 2008; Zhao et al., 2015; Shan et al., 2017), HIF1 $\alpha$ (Li et al., 2017; Han et al., 2018; Tang et al., 2018b), JNK (Zhai et al., 2017; Lin et al., 2018), PI3K/AKT (Zhang et al., 2015; Zhou et al., 2017; Ni et al., 2018; Tang et al., 2018b), STAT3 (Qiu et al., 2017; Tang et al., 2018a), p16INK4A and p73 (Jang et al., 2015), and PTEN (Nigorikawa et al., 2006; Chen et al., 2018; Zhang et al., 2018). These findings, at one hand, demonstrate that Shikonin can regulate various biological processes (Wang et al., 2019). On the other hand, they also illustrate a conundrum as to how exactly Shikonin regulates cellular processes and how such regulation contributes to the anticancer activity of Shikonin.

In order to understand how Shikonin elicits its anti-cancer activity, in the current study, we systematically investigated the effect of Shikonin on both the short-term proliferation and the long-term survival of various cancer cell lines originated from lung, breast, pancreas, colon and bone and one normal cell line derived from the liver. We use both chemical and genetic approaches to determine the involvement of cellular processes such as cell cycle, autophagy and apoptosis in the anti-cancer effect of Shikonin. Our data reveal that Shikonin simultaneously induces cell cycle arrest, cell death and autophagy, which collectively control cancer cell growth, survival and death.

\section{METHODS}

\section{Chemicals, Cell Culture and Reagents}

Shikonin (>99.0\%, \#517-89-5) and Z-VAD-FMK (\#S7023) were from Selleck Chemicals (Huston, TX, USA). Rapamycin (\#D9542) was purchased from Sigma (St. Louis, MO, USA). PEG300 (\#P815612) and Tween 80 (\#T818928) were purchased from Macklin Biochemical Co (Shanghai, China) or Selleck Chemicals (\#S6704 and S6702, respectively). Propidium iodide (PI, \# ICN19545810) was purchased from MP Biomedicals (Solon, OH, USA). Cell lines were grown in DMEM (U2OS,
PANC-1 and MDA-MB-231), RPMI-1640 (A549), or Ham's F12K medium (LO2) with 10\% FBS (ExCell bio, China) and $1 \%$ penicillin-streptomycin (Gibco/ThermoFisher, Franklin, $\mathrm{MA}, \mathrm{USA}$ ) at $37^{\circ} \mathrm{C}$ in $5 \% \mathrm{CO}_{2}$ and $98 \%$ humidity.

\section{Antibodies}

Antibodies for $\beta$-Actin (\#4970), p-mTOR (Ser-2448, \#5536), pERK1/2 (Thr-202/Tyr-204, \#4370), PARP (\#9542S), caspase-3 (\#9665S), anti-ULK1 (\#8054S), anti-pRSK (\#9344), anti-pATK (\#9275S), anti-BECN1 (\#3495S), cleaved Caspase-3 (\#9664S) and BAX (\#2772S), ERK1/2 (\#9102) and HRP-conjugated secondary anti-rabbit and anti-mouse antibodies (\#7076 and \#7074) were from Cell Signaling Technology (Beverly, MA, USA). Anti-P53 (\#SC-6243), anti-UHRF1 (\#SC-373750) and anti-P21 (\#SC-397) were from Santa Cruz Biotechnology (Santa Cruz, CA, USA). The anti-human LC3B (\#NB100-2220) and anti-P62 (\#NBP142821) antibodies were obtained from Novus Biologicals (Littleton, CO, USA). Anti-ATPA3 (\#ab2826) antibody was from Abcam (Cambridge, UK).

\section{Cell Proliferation and $\mathrm{IC}_{50}$ Determination}

Cell proliferation was determined by the cell counting kit-8 (cck8) assay (BeyotimeInst Biotech, China). Briefly, $5 \times 10^{3}$ A549 cells were seeded in each well of 96 -well plates, grown at $37^{\circ} \mathrm{C}$ for $24 \mathrm{~h}$, and treated with different concentrations of Shikonin for 1 , 2, 3 and 4 days. Then $10 \mu \mathrm{l}$ of cck- 8 solution was added to each well, incubated at $37^{\circ} \mathrm{C}$ for $2 \mathrm{~h}$ and the absorbance was determined at $570 \mathrm{~nm}$ from five replicates using a microplate reader (Synergy TM HT, BioTEK, USA).

To determine the $\mathrm{IC}_{50}$ of Shikonin, A549, PANC-1, MDAMB-131 and U2OS cancer cells, as well as LO2 liver fibroblasts, were treated with increasing dosages of Shikonin for $48 \mathrm{~h}$ and cell viability was determined as above-mentioned. Absorption in the blank well was subtracted and that in the DMSO control was set as $100 \%$, and others were normalized accordingly. The $\mathrm{IC}_{50}$ was calculated by the GraphPad Prism program.

\section{Cell Cycle Analysis}

For cell cycle analysis, $5 \times 10^{5}$ A549 or PANC- 1 cells were seeded in 6-cm dishes, treated with Shikonin for 0, 12 and $24 \mathrm{~h}$. Cells were harvested, washed once with cold PBS, fixed in $70 \%$ ethanol, washed with $\mathrm{PBS}$ once, re-suspended in $1 \mathrm{ml}$ of propidium iodide (PI) staining solution $(50 \mathrm{mg} / \mathrm{ml} \mathrm{PI}$ and 1 $\mathrm{mg} / \mathrm{ml} \mathrm{RNase}$ in sodium citrate buffer, $\mathrm{pH} 7.4$ ), incubated in the dark for $30 \mathrm{~min}$, and the cell cycle was measured by flow FACSCantoII cytometer (BD Biosciences, San Jose, CA, USA). Quantitation was performed using Multi-cycle Software (ModFit software) to determine the percentage of G1, S, G2/M and subG1 phase cells.

\section{Western Blotting}

Total cell lysates were harvested from A549, PANC-1, MDAMB-131, SW620, HT-29 or U2OS cells in lysis buffer (BeyotimeInst Biotech, China). The protein concentration was determined by the Pierce ${ }^{\circledR}$ BCA Protein Assay Kit (Pierce, \#23225, ThermoFisher, Franklin, MA, USA). Equal amount of total proteins $(\sim 40 \mu \mathrm{g})$ were separated on $6-10-15 \%$ 
SDS-PAGE, transferred to PVDF membranes (\#IPVH00010, Millipore/Sigma, Burlington, MA, USA), blocked with 5\% skim milk, probed with primary antibodies overnight at $4^{\circ} \mathrm{C}$, incubated with HRP-conjugated secondary antibodies for $1 \mathrm{~h}$ at room temperature, and imaged by the Tanon 5200 chemiluminescence imaging system (BioTanon, China).

\section{Annexin V Analysis}

Annexin $\mathrm{V}$ assay was conducted by the Annexin V fluorescein isothiocyanate (FITC) Apoptosis Detection Kit (KeyGEN BioTECH, Nanjing, China) according to manufacturer's instructions. Briefly, A549 cells were seeded in 6-well plates at a density of $1{ }^{*} 10^{6}$ cells/well in RPMI 1640 medium. The cells were treated or co-treated with $5 \mu \mathrm{M}$ Shikonin, $200 \mathrm{nM}$ CPT or both for $24 \mathrm{~h}$ at $37^{\circ} \mathrm{C}$ in $5 \% \mathrm{CO}_{2}$, collected and stained with Annexin V-FITC and propidium iodide (PI) in the dark at room temperature for $15 \mathrm{~min}$ and analyzed by flow cytometer (BD Biosciences, San Jose, CA, USA), and the results were analyzed using the software FACS Diva (BD Biosciences, San Jose, CA, USA).

\section{RNA Interference (RNAi)}

To generate ULK1 or BECN1 stably depleted cells, A549 or HeLa cells were stably infected with lentivirus shRNA vectors targeting these two genes, respectively. In brief, $6{ }^{*} 10^{6}$ HEK293T cells in $100 \mathrm{~mm}$ dish were transfected with virus packaging plasmid combinations containing $5 \mu \mathrm{g}$ individual shRNA lentivirus vector for ULK1 or BECN1, $2 \mu \mathrm{g}$ pMDL, $1 \mu \mathrm{g}$ VSVG and $1 \mu \mathrm{g}$ RSV-REV. After $48 \mathrm{~h}$, the supernatant was collected, centrifuged at $450 \mathrm{~g}$ for $5 \mathrm{~min}$ at room temperature, and added with fresh culture media (1:1 vol/vol) into pre-seeded A549 or HeLa cells in 6-well plates. Polybrene (final concentration at $4 \mu \mathrm{g} / \mathrm{ml}$ ) was added into the culture media $4 \mathrm{~h}$ later and incubated for $48-72 \mathrm{~h}$. The cells were then split into $100 \mathrm{~mm}$ dishes and added puromycin $(2-4 \mu \mathrm{g} / \mathrm{ml})$ to select stable cell clones. After around 10 days (or until the colonies formed), the cells were pooled and protein expression of ULK1 or BECN1 was confirmed by immunoblotting. shRNA vectors targeting ULK1 (\#TRCN0000000835 and \# TRCN0000000838) and BECN1 (\#TRCN0000033549 and \# TRCN0000033552) were obtained from Sigma (St. Louis, MO, USA).

\section{QPCR Analysis}

Total RNA was extracted from A549 cell cultures by the RNeasy plus kit (\#74134, Qiagen USA, Germany Town, MD, USA). The cDNA was synthesized using the Revert Aid first strand cDNA synthesis kit according to the manufacturer's procedure (\#K1622, ThermoFisher, Franklin, MA, USA). Real-time PCR was performed on a CFX96 Real-Time PCR system (Bio-Rad, Hercules, CA, USA) with SYBR Green Master Mix (\#208054, Invitrogen/ThermoFisher, Franklin, MA, USA). The mRNA level of target gene was determined by analyzing $2^{-\Delta \Delta \mathrm{Ct}}$ using HPRT1 as the internal control. The program used is: $95^{\circ} \mathrm{C}$ for $3 \mathrm{~min}$, followed by 40 cycles of $95^{\circ} \mathrm{C} 10 \mathrm{~s}$ and $60^{\circ} \mathrm{C} 30 \mathrm{~s}$. Immediately following the cycle, melt curve is determined by heating the sample to $95^{\circ} \mathrm{C}$ for $10 \mathrm{~s}$, reducing to $60^{\circ} \mathrm{C}$ for $30 \mathrm{~s}$, and then gradually increasing to $95^{\circ} \mathrm{C}$ with $0.5^{\circ} \mathrm{C}$ increment increase.
Primers for RT-PCR are:

TP53-F: 5'-CTTCCATTTGCTTTGTCCCG-3'

TP53-R: 5'-CATCTCCCAAACATCCCTCAC-3'

CDKN1A (P21)-F: 5' -AACTAGGCGGTTGAATGAGAG-3'

CDKN1A (P21)-R: 5'-GAGGAAGTAGCTGGCATGAAG-3'

c-FOS-F: 5'-CTCAAGTCCTTACCTCTTCCG-3'

c-FOS-R: 5'-GAGAAAAGAGACACAGACCCAG-3'

JUN-F: 5' -TGTCCGAGAACTAAAGCCAAG-3'

JUN-R: 5' -TCAATGTTAACGAAAAGTCCAACG-3'

ATG3-F: 5'-GATGGCGGATGGGTAGATACA-3'

ATG3-R: 5' -TCTTCACATAGTGCTGAGCAATC-3'

ATG5-F: 5'-AAAGATGTGCTTCGAGATGTGT-3'

ATG5-R: 5'-CACTTTGTCAGTTACCAACGTCA-3'

HPRT1-F: 5' -AGCTTGCTGGTGAAAAGGA-3'

HPRT1-R: 5' -CCAAACTCAACTTGAACTCTCATC-3'

\section{Clonogenic Cell Survival Assay}

Clonogenic survival assay was used to determine the long-term survival capability of cells. Briefly, after treatment, 5000 A549, PANC-1, U2OS, MDA-MB-231 or LO2 cells were seeded into 6well plates in triplicate and cultured in drug-free full media for 10-14 days or until cell colonies were clearly visible. The culture medium was changed every 3 days. Cells were washed once with phosphate buffered saline and fixed in acetic acid-methanol solution $(1: 7, \mathrm{vol} / \mathrm{vol})$ at room temperature for $5 \mathrm{~min}$. After staining with $0.1 \%$ crystal violet dye in methanol at room temperature for $15 \mathrm{~min}$, the plates were then gently rinsed under tap water, placed upside down to allow air dry. The dried plates were first scanned, then dissolved in 1\% SDS. The plates were placed on a shaker to incubate until no areas of dense coloration were visible at the bottom of wells. Finally, the absorbance of each well was read at $570 \mathrm{nM}$ using a microplate reader (Synergy TM HT, BioTEK, USA).

\section{Mouse Studies}

For mouse studies, 5-6 weeks old female BALB/c Nu/Nu mice were purchased from Beijing HFK Bioscience CO. LTD (Beijing, China) or the Jackson Laboratory (Bar Harbor, ME, USA). All mice were housed in-group in cages with bedding, controlled temperature $\left(23 \pm 2^{\circ} \mathrm{C}\right)$, humidity $(50 \pm 5 \%)$ and illumination ( $12 \mathrm{~h}$ light/dark cycle). Mice were adapted to the facility for 1 week before experiments. All animal experiments were performed in accordance with the National Institutes of Health's Guide for the Care and Use of Laboratory Animals (NIH publication No. 80-23, revised in 1996) and were approved by the Institutional Animal Care and Use Committee at Jinan University or Case Western Reserve University. In addition to all procedures to be sterile, mice were allowed access to sterile food and water and libitum. Shikonin was prepared at 140 or $70 \mathrm{mM}$ stock solution in DMSO. The injection solution was always freshly prepared by mixing the stock solution with PEG300 (30\%), Tween-80 (5\%) and sterile $\mathrm{H}_{2} \mathrm{O}$ (various based on the Shikonin concentration). 
The solution is stable for at least 4 hours at room temperature, which is sufficient for injection.

For animal toxicity experiment, mice were injected with 20, 10, 5,3 and $1 \mathrm{mg} / \mathrm{kg}$ Shikonin i.p. twice a week for 2 weeks. Animal survival was documented and plotted by Kaplan-Meier survival curve. For xenografted tumor study, $5 \times 10^{6}$ A549 cells suspended in RPMI-1640 medium without serum were injected subcutaneously into the right flank of each mouse. Tumors were allowed to grow till the volume reached approximately $\sim 100 \mathrm{~mm}^{3}$, and mice were randomly divided into the following two groups with 6 mice in each group: (1) Control; and (2) Shikonin (2 mg/kg). Shikonin was given by i.p. twice a week. Tumor volume and body weight were measured at least twice a week for 3 weeks. Tumor volume was calculated using the formula $\mathrm{V}=\left(\mathrm{L} \times \mathrm{W}^{2}\right) \times 0.52$ where $\mathrm{V}=$ volume, $\mathrm{L}=$ length, $\mathrm{W}$ = width.<smiles>CC(C)=CC[C@@H](O)C1=CC(=O)c2c(O)ccc(O)c2C1=O</smiles>

FIGURE 1 | Chemical structure of Shikonin.

\section{Statistical Analysis}

All cell culture experiments were performed at least twice. Data are presented as mean \pm standard deviation. The statistical analysis was conducted by the Prism 5.0 software. Pairwise comparison was performed using a two-tailed Student $t$-test, whereas one-way ANOVA was used to compare multiple comparisons. $P$-values of less than at least 0.05 were considered statistically significant.

\section{RESULTS}

\section{Shikonin Displays a Broad Inhibitory Effect on Cancer Cell Growth}

To understand the anti-cancer mechanisms of Shikonin (Figure 1), we first evaluated the $\mathrm{IC}_{50}$ of this compound in four different cancer cell lines including lung adenocarcinoma (A549), triple-negative breast cancer (MDA-MB-231), pancreatic cancer (PANC-1), and osteosarcoma (U2OS). We reason that an evaluation of the effect of Shikonin on these cancer cell lines may provide insights into the preclinical development of this agent in the future. The results show that Shikonin treatment for $48 \mathrm{~h}$ displayed an $\mathrm{IC}_{50}$ at around $1-2 \mu \mathrm{M}$ in all cancer cell lines tested (Figures $\mathbf{2 A - D}$ ). On the other hand, the $\mathrm{IC}_{50}$ of Shikonin in a normal human hepatocyte LO2 cell line is roughly 4 -fold higher than that
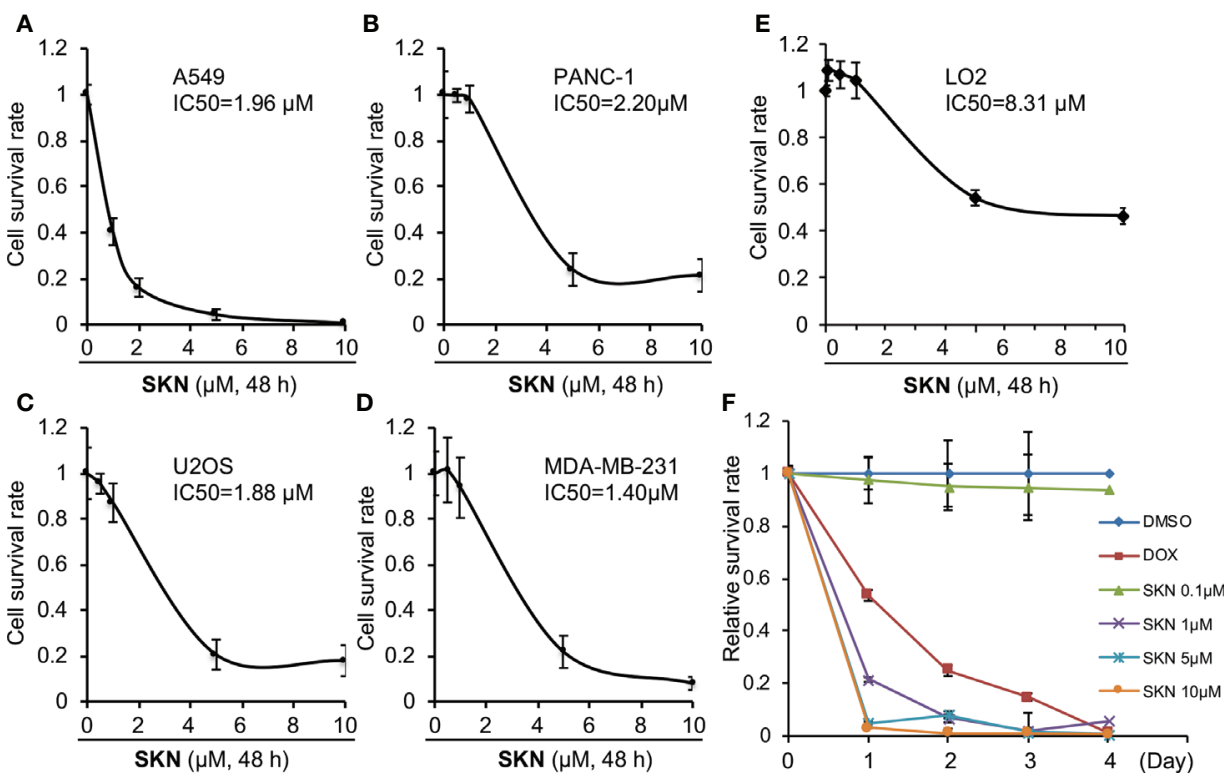

FIGURE 2 | Effects of Shikonin on cancer cell growth. A549 (A), PANC-1 (B), U2OS (C), MDA-MB-231 (D), or LO2 (E) cells were treated with indicated concentrations of Shikonin for $48 \mathrm{~h}$, and cell survival was analyzed by the CCK-8 assay. Data were normalized to that of DMSO control and presented as relative survival rate from five replicates. $\mathrm{IC}_{50}$ values were analyzed using the GraphPad software. (E) A549 cells were treated with DMSO or different concentrations of Shikonin or $10 \mu \mathrm{M}$ doxorubicin (DOX) for 1, 2, 3 and 4 days, and cell survival was analyzed by the CCK-8 assay. The absorbance of Shikonin-treated group was normalized to that of the same day DMSO control, which yields the relative survival rate. Data represent mean and standard deviation from five replicates. (F) A549 cells were treated with DMSO or different concentrations of Shikonin for 1, 2, 3 and 4 days, and cell survival was analyzed by CCK-8 assay. The absorbance of Shikonin-treated group was normalized to that from the same day DMSO control, which yields the relative survival rate. Data represent mean and standard deviation from five replicates. 
in cancer cells (Figure 2E). To further probe the growth inhibitory effect of Shikonin, we performed a real time cell proliferation assay using A549 as the representative cell line. The results show that Shikonin both dose- and timedependently suppressed cancer cell proliferation (Figure 2F). At a dose at or greater than $1 \mu \mathrm{M}$, Shikonin showed even more growth inhibitory effect than a chemotherapeutic drug doxorubicin (Figure 2F), indicating a strong anti-cancer effect of Shikonin.

Then to determine the impact of Shikonin on long-term cell survival, we performed a dose-dependent clonogenic survival assay in A549 and PNAC-1 cancer cells. The results show that Shikonin dose-dependently inhibited the long-term survival of both cancer cell lines (Figures 3A, B). Similar inhibitory effects were observed in MDA-MD-231 and U2OS cancer cells (Figure 3C). On the other hand, Shikonin did not inhibit the long-term survival of the normal LO2 cell (Figure 3C), even though it also inhibited the short-term cell proliferation albeit at a much lesser degree than cancer cells (Figure 2E). Together, these data demonstrate a general anti-cancer effect of Shikonin and suggest that it has much less inhibitory effect on normal cells. These findings also allow us to choose selected cell lines from these four types to perform subsequent studies in order to avoid redundancy.

\section{Shikonin Induces G2/M Phase Cell Cycle Arrest and Robust Cell Death}

To understand how Shikonin inhibited cancer cell growth, we first monitored the cell cycle profile in the presence or absence of Shikonin. Flow cytometry data revealed that Shikonin induced a time-dependent accumulation of cells at the G2/M phase in A549 and PANC-1 (Figures 3D, E) cells, suggesting that Shikonin induces cell cycle arrest at the G2/M phase.

To understand the molecular basis underlying the growth inhibitory effect of Shikonin, we conducted immunoblotting to examine expression of genes involved in cell growth, cell death and autophagy by Shikonin. We found that treatment of A549 cells with Shikonin time-dependently increased the cleavage of poly (ADP-ribose) polymerase (PARP) and caspase 3 (cCasp3) (Figure 4A), two known apoptotic cell death markers (Fernandes-Alnemri et al., 1994; Nicholson et al., 1995; Duriez and Shah, 1997). These findings are in general agreement with previous reports (review (Wang et al., 2019)). To confirm that Shikonin indeed induced cell death,
A
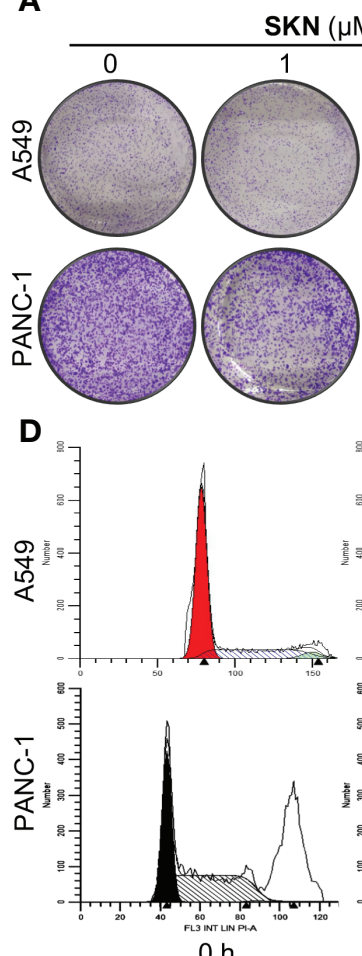

$\mathrm{Oh}$
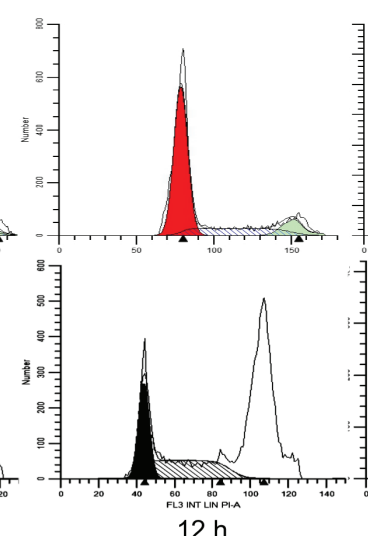

B
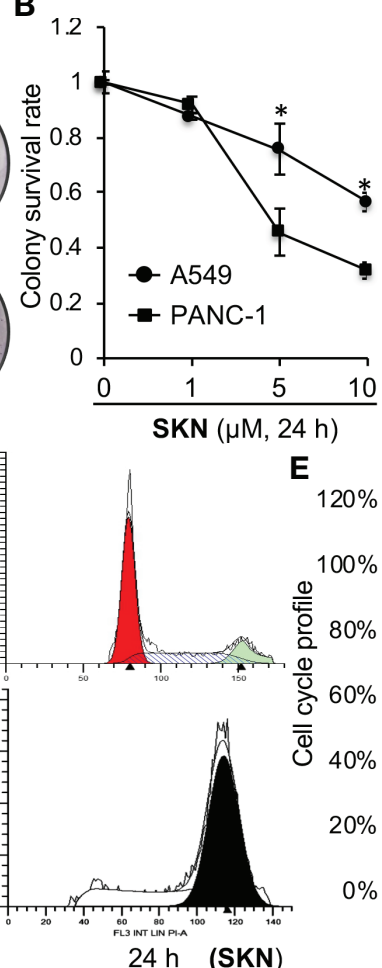

C
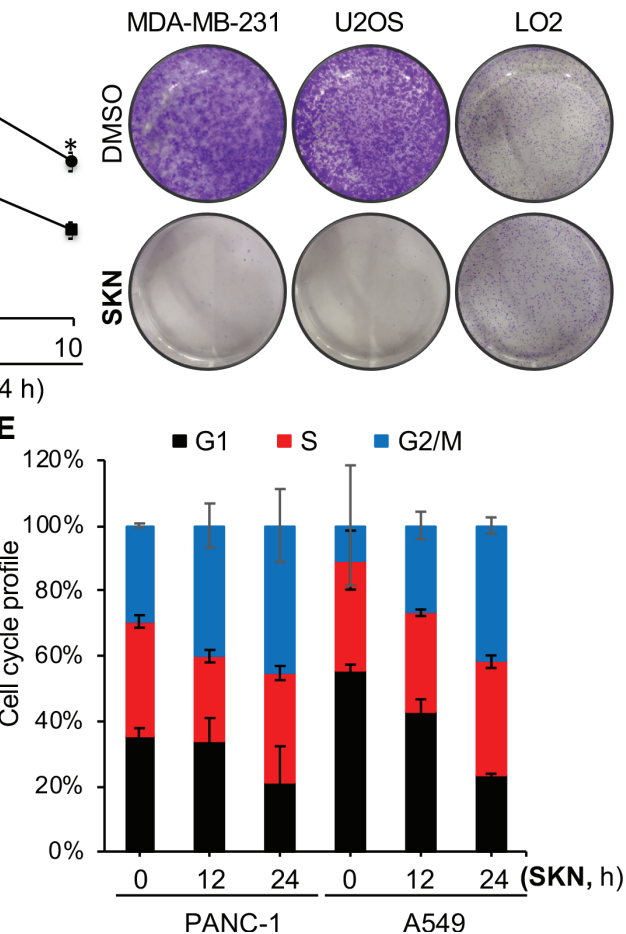

FIGURE 3 | Effects of Shikonin on cell survival and cell cycle. (A) A549 or PANC-1 cells were treated with increasing concentrations of Shikonin for 24 h, and clonogenic survival assay was measured. Representative images are shown. (B) Quantitation of relative survival from (A). Data represent mean and standard deviation from five replicates. ${ }^{*} \mathrm{P}<0.001$ compared with the DMSO control. (C) MDA-MB-231, U2OS or LO2 cells were treated with $5 \mu$ M of Shikonin for 24 h, and clonogenic survival assay was measured. Representative images are shown. (D) A549 or PANC-1 cells were treated with $5 \mu \mathrm{M}$ of Shikonin for 0, 12 and 24 h, fixed and cell cycle profile was analyzed. (E) Quantitation of cell cycle distribution for A549 and PANC-1 cells from $\boldsymbol{D}$. Data represent average and standard deviation from two independent experiments. 

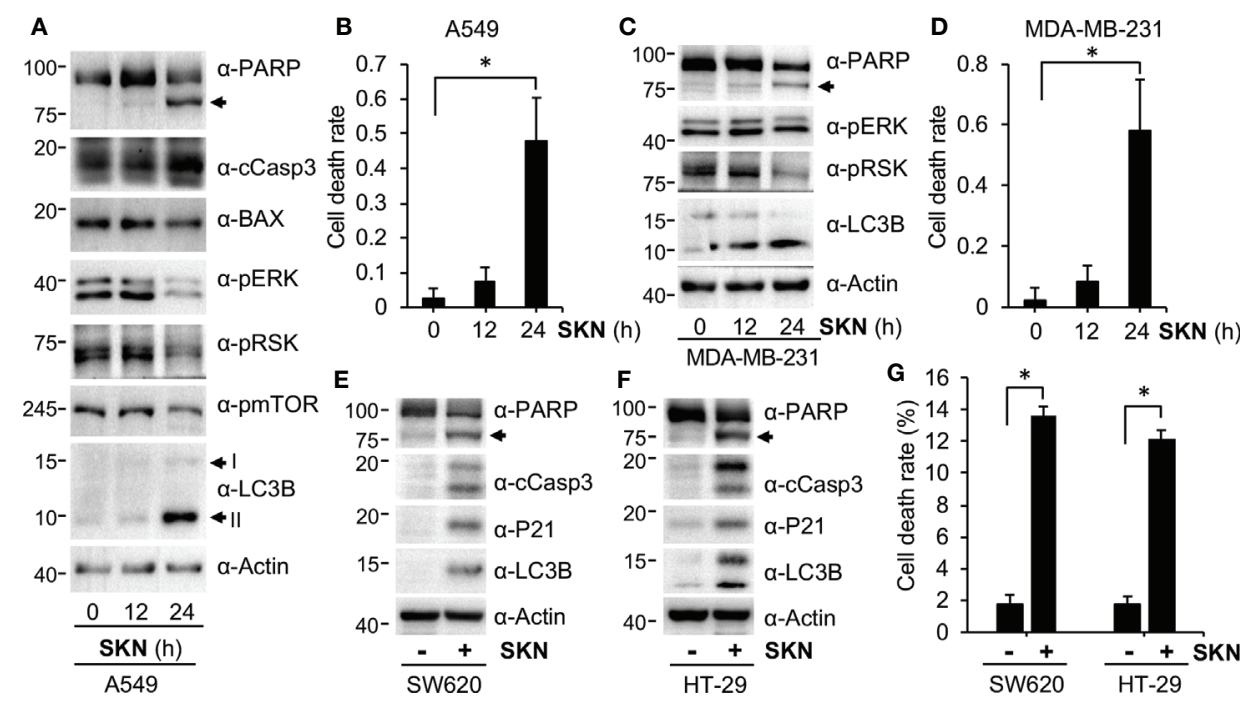

FIGURE 4 | Shikonin induces cell death. (A) PANC-1 cells were treated with $5 \mu \mathrm{M}$ of Shikonin for 0,12 and $24 \mathrm{~h}$, and protein expression was measured using specific antibodies. I and II indicate non-modified and lipidated forms of LC3B, respectively. Anti-PARP recognizes both full-length and cleaved PARP proteins, and the latter is indicated by the arrow. (B) Quantitation of dead cell population from cells in (A) by trypan blue staining. Data represent mean and standard deviation from five replicates. *P $<0.001$. (C) MDA-MD-231 cells were treated with $5 \mu \mathrm{M}$ of Shikonin for 0,12 and $24 \mathrm{~h}$, and protein expression was measured by specific antibodies. (D) Quantitation of dead cell population from $\boldsymbol{C}$ by trypan blue staining. Data represent mean and standard deviation from five replicates. ${ }^{*} P<0.001$. SW620 (E) or HT-29 (F) cancer cells were treated with $5 \mu \mathrm{M}$ of Shikonin for $12 \mathrm{~h}$, and protein expression was measured by specific antibodies. (G) Quantitation of dead cell population from $\boldsymbol{E}$ and $\boldsymbol{F}$ by trypan blue staining. Data represent mean and standard deviation from five replicates. ${ }^{*} \mathrm{P}<0.001$.

we measured dead cell population using trypan blue staining. The results show that Shikonin induced significant cell death induction after $24 \mathrm{~h}$ treatment (Figure 4B). Similar results were obtained from breast MDA-MB-231 cancer cells (Figures 4C, D) and colorectal SW620 and HT-29 cancer cells (Figures 4E-G). Except A549, PANC-1, MDA-MB-231, SW620 and HT-29 are all P53 mutant cancer cell line. MDAMB-231 carries a homozygous c.839G > A (R280K) mutation, whereas PANC1 is homozygous for c.818G > A (R273H). On the other hand, SW620 carries both R273H and P309S mutations whereas HT-29 is a R273H P53 mutant cancer cell line. Hence, these results demonstrate that Shikonin induces cell death in both P53 wild type and mutant cancer cell lines.

\section{Shikonin Inhibits the ERK Growth Signal Pathway}

Shikonin had been previously reported to both inhibit and activate the extracellular signal-regulated kinas (ERK) pathway, providing inconsistent results of Shikonin (review (Wang et al., 2019)). During analyses, we observed that Shikonin timedependently inhibited phosphorylation of ERK in both A549 and MDA-MB-231 cells (Figures $\mathbf{4 A}, \mathbf{C}$ ), indicating that Shikonin inhibited the ERK signaling. In agreement with the inhibition in phosphorylation of ERK, phosphorylation of the ERK downstream factor RSK was also reduced by Shikonin in a time-dependent manner (Figures $\mathbf{4 A}, \mathrm{C}$ ). These results suggest that Shikonin inhibits, but not activates, the ERK pathway, which may lead to the cell growth inhibitory effect of Shikonin.

\section{Shikonin Mainly Induces Apoptotic Cell Death}

To confirm the role of apoptosis in Shikonin-induced cell death, we first carried out Annexin V staining. The FACS results show that Shikonin time-dependently induced a moderate increase in the apoptotic cell population (Figure 5A), suggesting the activation of apoptosis by Shikonin. However, Annexin V staining illustrated a much weaker activity of Shikonin than that of PARP cleavage or trypan blue staining. Such a relatively weak effect of Annexin V might be due to the insensitivity of the assay in measuring dead cells in this particular experimental setting, as well as the involvement of other cell death mechanisms.

To answer this question and to confirm the involvement of apoptosis, we assessed the effect of a pan apoptosis inhibitor, ZVAD-FMK (Z-VAD), on Shikonin-induced cell death. The results show that Z-VAD co-treatment dramatically reduced the levels of cleaved PARP or Caspase 3 induced by Shikonin (Figure 5B). Consistently, Z-VAD significantly reduced the dead cell population of both A549 and PNAC- 1 cells measured by trypan blue staining in the presence of Shikonin (Figure 5C). We realize that the effect of ZVAD was weaker in PANC-1 cells than in A549 cells and that it did not completely block Shikonin-induced cell death (Figure 5C), suggesting that other types of cell death such as necroptosis might also be involved (review (Wang et al., 2019)).

\section{Shikonin Activates the Protective Autophagy Pathway}

Cells often activate the protective autophagy pathway to survive when encountering stressful situations, for instance, in the 

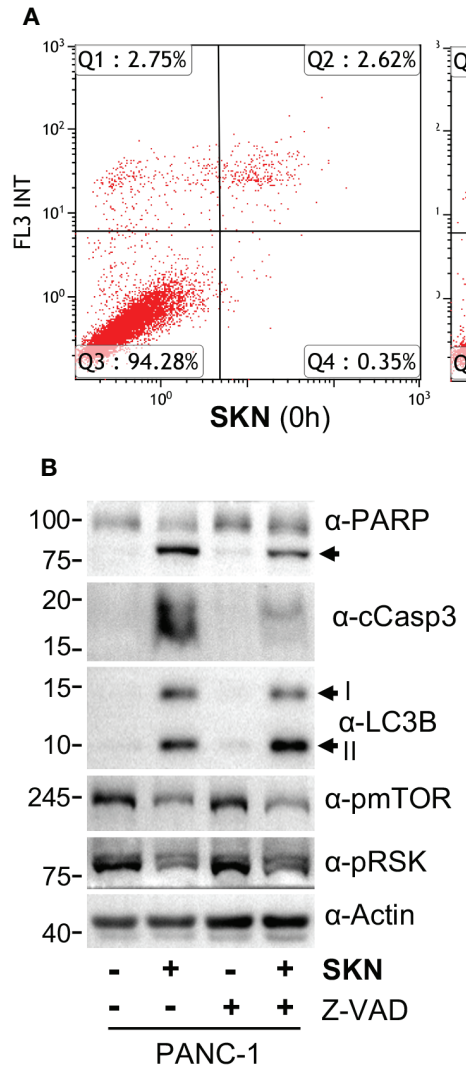

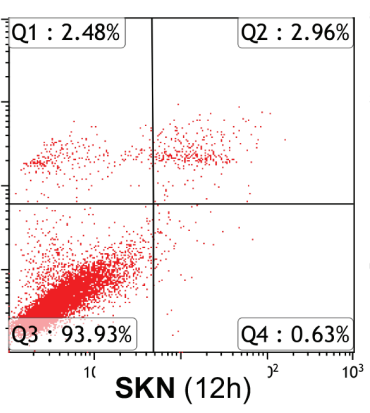

C

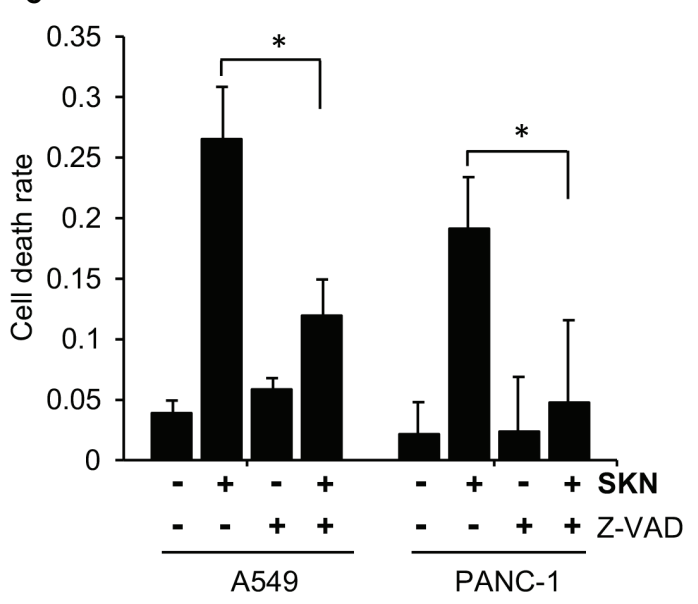

FIGURE 5 | Apoptosis-dependent cell death induction by Shikonin. (A) A549 cells were treated with $5 \mu \mathrm{M}$ of Shikonin for 0,12 and $24 \mathrm{~h}$, and apoptotic cells were analyzed by Annexin V staining. (B) PANC-1 cells were treated with $5 \mu \mathrm{M}$ of Shikonin in the presence or absence of $10 \mu \mathrm{M} Z$-VAD for $24 \mathrm{~h}$, and protein expression was measured by specific antibodies. Cleaved PARP, LC3B forms I and II are indicated by arrows. (C) Quantitation of dead cell population from $\boldsymbol{B}$ by trypan blue staining. Data represent mean and standard deviation from five replicates. ${ }^{*} \mathrm{P}<0.001$.

absence of nutrient or energy supply (Kundu and Thompson, 2008; Levine and Kroemer, 2008). Consistent with this idea and previous reports (review (Wang et al., 2019)), we found that Shikonin greatly increased the level of the lipidated form of LC3B (i.e., LC3B II in Figures 4A, B), a well-known marker for autophagy activation (Kundu and Thompson, 2008; Mizushima and Komatsu, 2011). These results led us to further determine the role of autophagy in Shikonin-induced cellular response. To this end, we decided to inhibit autophagy by depleting critical autophagic genes including Unc-51 like autophagy activating kinase 1 (ULK1) and Beclin 1 (BECN1) by RNA interference (RNAi). ULK1 is a serine/threonine protein kinase that plays a critical role in the initiating step of autophagy (Matsuura et al., 1997; Kamada et al., 2000; Wirth et al., 2013). We generated an A549 cell line with ULK1 stably reduced (Figure 6A). We found that inhibiting ULK1 expression further increased the levels of cleaved Caspase 3 and PARP caused by Shikonin (Figure 6A), as well as elevated Shikonininduced cell death (Figure 6B). BECN1, by forming a complex with the class III type phosphatidylinositol 3 kinase VPS34, is another key autophagy initiator (Funderburk et al., 2010). Therefore, we stably depleted BECN1 in HeLa cells and observed that even a partial depletion of BECN1 further elevated the level of cleaved PARP (Figure 6C) and cell death by Shikonin (Figure 6D), reinforcing the idea that autophagy is activated during Shikonin treatment. We noticed that Shikonin did not clearly induced LC3B lipidation in HeLa cells (Figure 6C). The reason is unclear at this moment; however, this could be a cell line specific effect as we constantly observed the conversion of LC3B in A549, PNAC-1, MDA-MB-231, SW620 and HT-29 cells. Overall, these results suggest that Shikonin generally activates autophagy and such autophagy activation helps to counteract the cell death-inducing effect of Shikonin.

\section{Shikonin Regulates Expression of Genes Involved in Cell Growth, Autophagy and Cell Cycle}

Next we intended to further understand the molecular mechanisms by which Shikonin inhibited cell cycle progression, activated autophagy and induced cell death. We found that Shikonin treatment greatly increased the protein level of P21 in A549 (Figure 7A), MDA-MB-231 (Figure 7B), PNAC1 (Figure 7C) and U2OS cells (Figure 7D), as well as in SW620 (Figure 4E) and HT-29 (Figure 4F) cells. Shikonin had also been previously reported to induce P21 expression in breast cancer 

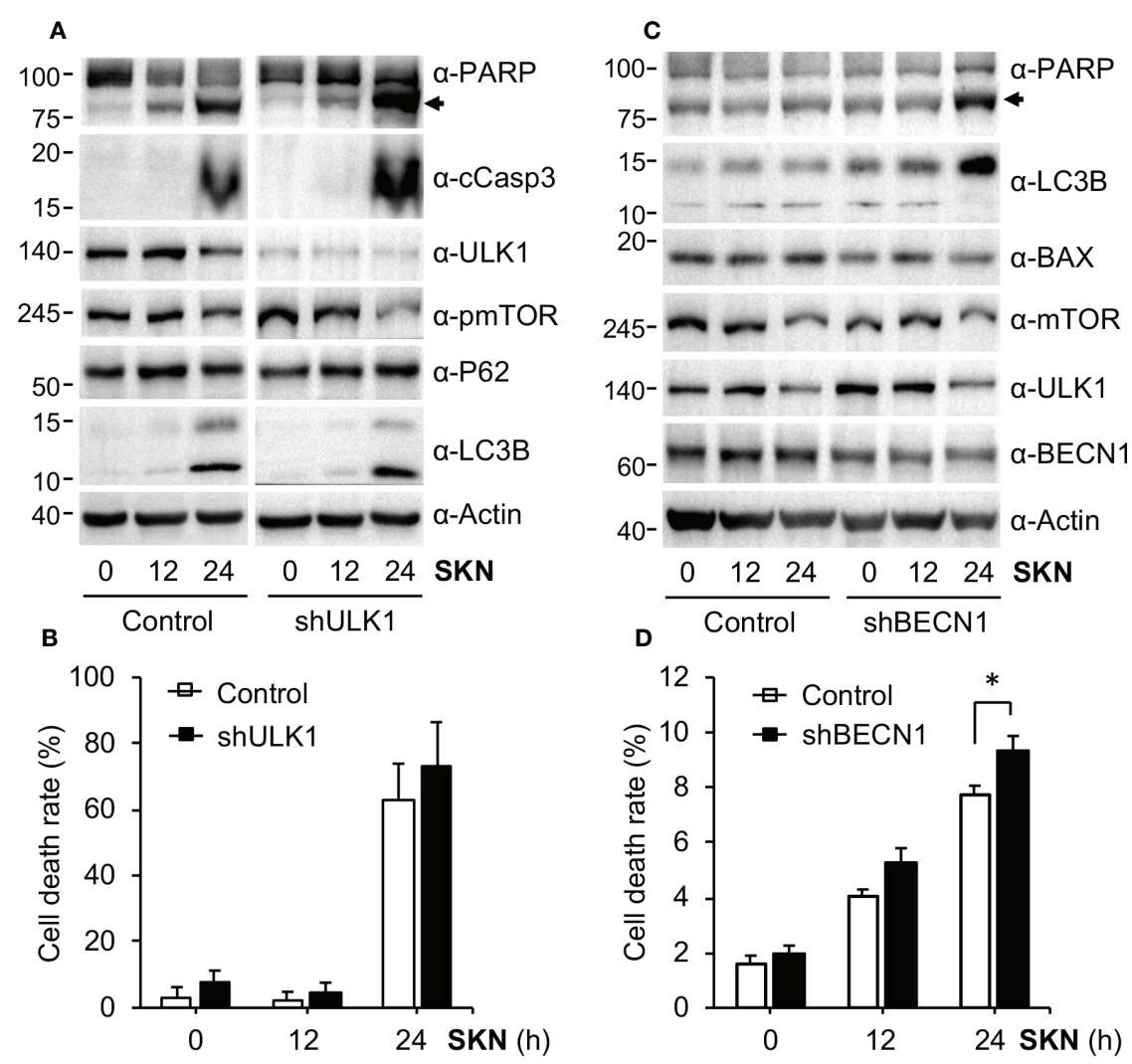

FIGURE 6 | Shikonin activated autophagy. (A) A549 control or ULK1 depleted cells were treated with $5 \mu \mathrm{M}$ of Shikonin for 0 , 12 and 24 h, and protein expression was measured. (B) Quantitation of dead cell population from (A) by trypan blue staining. Data represent mean and standard deviation from five replicates. (C) HeLa control or BECN1 stably depleted cells were treated with $5 \mu \mathrm{M}$ of Shikonin for 0, 12 and $24 \mathrm{~h}$, and protein expression was measured using specific antibodies. (D) Quantitation of dead cell population from $\mathbf{C}$ by trypan blue staining. Data represent mean and standard deviation from five replicates. ${ }^{*} \mathrm{P}<0.05$.

cells (Zhang et al., 2013; Zheng et al., 2018). P21 is a potent inhibitor of cyclin-dependent kinases and induces cell cycle arrest in G1 or G2/M phase (Xiong et al., 1993; Abbas and Dutta, 2009; Karimian et al., 2016; Kastenhuber and Lowe, 2017). Hence, the upregulation in P21 is consistent with the G2/M phase cell cycle arrest by Shikonin. These data suggest that Shikonin induced upregulation in P21 in both P53 wild type and mutant cancer cell lines.

To confirm these results, we performed quantitative PCR (qPCR) to determine the mRNA level of CDKN1A, the gene encoding P21. The results show that Shikonin robustly increased CDKN1A levels in A549 cells in a time-dependent fashion (Figure 7E). On the other hand, Shikonin initially slightly increased TP53 (the gene encoding P53) transcription followed by a reduction of it to the basal level (Figure 7F). The difference in transcription between CDKN1A and TP53 again supports the idea that Shikonin-upregulated P21 is unrelated to P53, which is consistent with previous reports showing that P21 transcription can be both P53-dependent and -independent (Hoffman et al., 2002; Kastenhuber and Lowe, 2017).

To determine how Shikonin induced cell death and autophagy activation, we also assessed mRNA levels of critical genes involved in these pathways. We found that Shikonin time-dependently inhibited the expression of growth-promoting genes such as JUN and $c$-FOS (Figures 7G, $\mathbf{H})$, as well as increased the levels of autophagy genes like ATG3 and ATG5 (Figures 7I, J).

\section{Shikonin Inhibits Tumor Growth in Mice}

To determine if the strong in vitro anticancer effect of Shikonin can be recapitulated in vivo, we decided to perform mouse xenograft experiment. First, we assessed the toxicity of Shikonin in mice. We found that i.p. injection of Shikonin at a dose of $10 \mathrm{mg} / \mathrm{kg}$ and above caused acute death of mice, whereas a dose at $5 \mathrm{mg} / \mathrm{kg}$ induced partial death of the animal after 2 weeks of injection (Figure 8A). In contrast, a dose of 1-3 mg/kg did not result in mouse death (Figure 8A) despite slight weight loss of mice after 2 weeks of drug administration (Figure $\mathbf{8 B}$ ).

Given the general anticancer effect of Shikonin observed in various cancer cell lines, we chose to use the A549 lung adenocarcinoma cell line as the representative to perform the in vivo study. To this end, we inoculated A549 cancer cells into nude mice subcutaneously, and examined the effect of Shikonin on tumor growth. Our data show that Shikonin significantly 


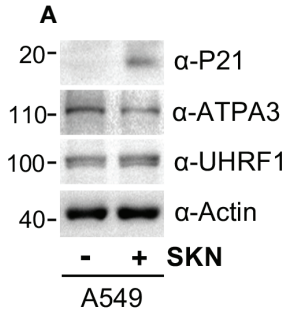

E
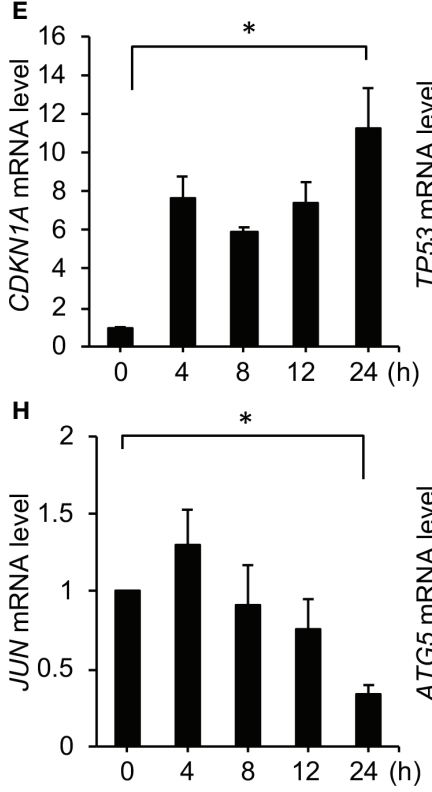

B

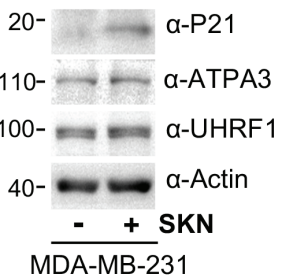

F
C

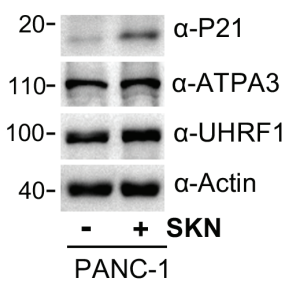

D

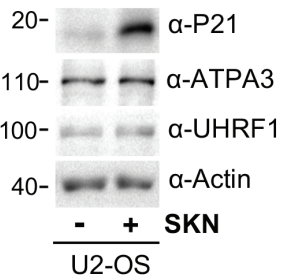

G

(1)

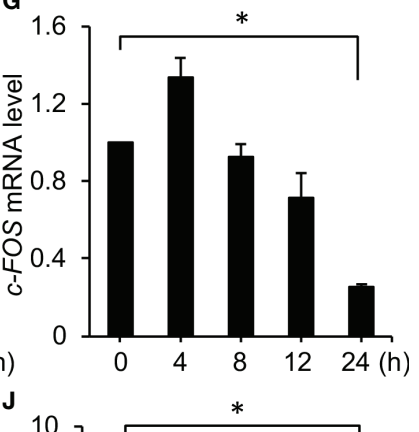

(h)

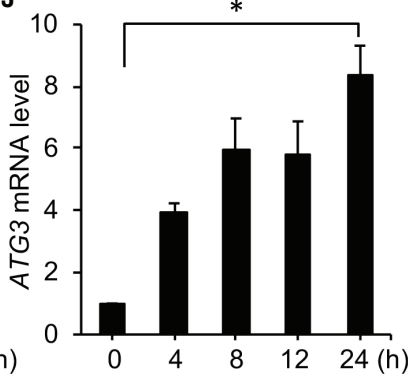

FIGURE 7 | Shikonin upregulates P21 and alters gene transcription. (A) A549, (B) MDA-MB-231, (C) PANC-1 and (D) U2OS cells were treated with $5 \mu$ M of Shikonin for $6 \mathrm{~h}$, and protein expression was measured by specific antibodies. For qPCR analysis, A549 cells were treated with $5 \mu \mathrm{M}$ Shikonin for 0 , 4,8 , 12 and 24 h, RNA was isolated, and expression of genes including CDKN1A (E), TP53 (F), C-FOS (G), JUN (H), ATG5 (I), and ATG3 (J) was analyzed. Data represent mean and standard deviation from five replicates. ${ }^{*} \mathrm{P}<0.001$ between 0 and $24 \mathrm{~h}$ groups.

suppressed tumor growth in mice (Figure 8C), confirming the anticancer effect of this compound in vivo.

\section{DISCUSSION}

It has been shown that Shikonin elicits a wide variety of biological activities including the heavily studied anticancer effect (Wang et al., 2019). Yet, the underlying molecular mechanisms remained unclear. Studies have shown that Shikonin could inhibit cell proliferation, induce cell death and activate autophagy, pointing to complicated biological outcomes that often depend on the cellular context including cell types, treatment conditions and assay methods (Wang et al., 2019). In the current study, we presented evidence to show a broad anticancer effect of Shikonin that is largely consistent with previous reports. However, compared with previous reports, our studies are novel in a number of ways.

One of the major reasons for the uncertainty of the anticancer mechanism of Shikonin is that usually one type of cancer cell line was used and only short-term cell culture effect was measured using assays like MTT. Further, there is the lack of well-planned genetic and pharmacological studies to determine the time course of biological processes caused by Shikonin. Here, we systematically investigated the effect of Shikonin on both short-term and long-term growth of four different cancer cell lines and one normal cell line from the liver. In determining cell death (using trypan blue exclusion and Western blotting tools), two additional colorectal cancer cell lines were included to confirm the generality of Shikonin-induced cell death and autophagy activation. Our results reveal that Shikonin simultaneously inhibits cell cycle, induces cell death and activates autophagy. Although Shikonin had been reported to have effects on these biological processes, as far as we know, we are the first to show that Shikonin could do all of these at the same time in various cancer cell lines. These results clearly point out the complexity of the biological effect of Shikonin, as well as highlight a broad spectrum of activities of this compound.

Our studies advanced out understanding of cellular mechanisms underlying Shikonin-induced cell cycle arrest and cell death. Here we present strong evidence acquired from six different cancer cell lines from different tissues and organs to 

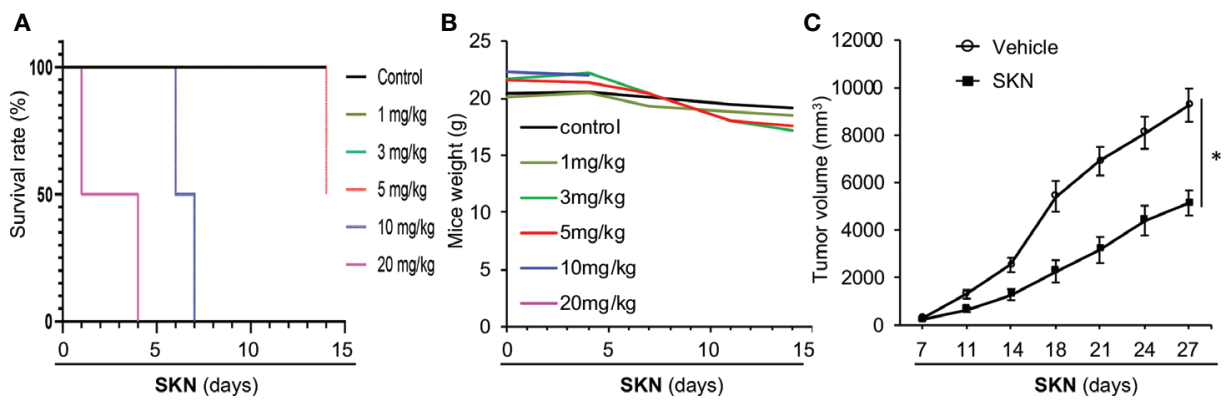

FIGURE 8 | Mouse studies of Shikonin. (A) 5-6 week old nude mice $(n=2)$ were injected with vehicle control or 1, 3, 5, 10 or 20 mg/kg Shikonin i.p. twice a week for 2 weeks, and mouse survival was plotted by the Kaplen-Mier plotter. (B) Body weight of mice in (A) was recorded and plotted over time. (C) For nude mice tumor study, $5 * 10^{6}$ A549 cells were inoculated into nude mice subcutaneously. When the tumor reached around $100 \mathrm{~mm}^{3}$ in size, the mice were treated with 2 $\mathrm{mg} / \mathrm{kg}$ of Shikonin i.p. twice a week for 3 weeks. Tumor volumes were measured during this time period. Data represent mean and standard error of tumor volume from six mice per group. ${ }^{*} \mathrm{P}<0.001$.

show that Shikonin arrested the cell cycle at the G2/M phase likely due to its up-regulation of $\mathrm{P} 21$, a potent cell cycle inhibitor that arrests cells at G1 or G2/M phase (Xiong et al., 1993; Abbas and Dutta, 2009; Karimian et al., 2016; Kastenhuber and Lowe, 2017). Such an increase in P21 was accompanied with elevated transcription. Interestingly, the increases in both P21 and cell death induced by Shikonin were observed in both P53 wild type and mutant cancer cell lines, highly supporting P53 statusindependent activities of Shikonin.

Our studies also helped to clear some confusion about Shikonin in the literature. First, autophagy activation had been presented as both a positive and a negative factor for Shikonin-induced cell death. For instance, Kim et al. reported that autophagy activation protected cells from Shikonininduced necroptosis (Kim et al., 2017), whereas another study suggested that autophagy activation contributed to Shikonininduced cell death (Zhu et al., 2019). Here we also confirmed autophagy activation by Shikonin in a wide range of cancer cell lines except HeLa. We further showed that autophagy protected, but not promoted, Shikonin-induced cell death, illustrating a protective effect of autophagy in the presence of Shikonin. Nonetheless, the effect of autophagy activator or autophagic gene depletion was generally weak, indicating that autophagy activation in the presence of Shikonin may merely represent a consequence of cellular reaction to stress (in this case, Shikonin treatment)(Wang et al., 2019). Second, the ERK kinase belongs to the MAPK family kinase and plays a critical role in regulating cell growth (Peti and Page, 2013). Not surprisingly, Shikonin has been shown to inhibit the ERK kinase, contributing to its growth inhibitory effect. However, a number of studies also reported that Shikonin activated ERK (Wu et al., 2005; Chang et al., 2010; Shen et al., 2012; Han et al., 2019) and such activation seemed to promote the cell death inducing effect of Shikonin (Zhuang and Schnellmann, 2006). Therefore, the effect of Shikonin on the ERK pathway remained controversial. Here, we show that Shikonin reduced the levels of activated ERK and its downstream factor RSK in multiple cancer cell lines, indicating that Shikonin inhibits the ERK pathway at least under these particular experimental conditions. qPCR results showed a reduction in the expression levels of growth-related genes, which is consistent with the data showing the inhibition of the ERK pathway. These results may help to clear the confusion as to the role of the ERK signal in the effect of Shikonin.

Also we, for the first time, provided a detailed toxicity analysis of Shikonin in nude mice. Our studies reveal a relatively low dose tolerance of Shikonin in mice $(<3 \mathrm{mg} /$ $\mathrm{kg}$ ). As to why previous studies reported even greater than 10 $\mathrm{mg} / \mathrm{kg}$ dose of Shikonin in mice, we speculate that it was largely due to inappropriate solution making of Shikonin in previous studies. We have tested various solvents to allow stable dissolving of Shikonin. We found that the Shikonin solution can be stable for as long as $4 \mathrm{~h}$ at room temperature only when dissolved in PEG300 (30\%), Tween-80 (5\%) and sterile $\mathrm{H}_{2} \mathrm{O}$. Otherwise, Shikonin seemed to be dissolved initially but will precipitate within $30 \mathrm{~min}$, preventing the injection of precise amount of chemicals into mince. Consequently, that led to the incorrect impression that mice can tolerate high dose of Shikonin. This additional analysis allowed us to precisely control the amount of Shikonin and determine its toxicity in mice. By combining with the effect on normal cell line, we believe that we can lower the dose of Shikonin in future studies. Together, our results demonstrate strong anticancer effect of Shikonin across a wide range of human cancer cell lines and reveal activation of biological pathways that contributed to the growth inhibitory effect of Shikonin. These results will shed significant light on our understanding of this molecule in cancer therapy.

\section{DATA AVAILABILITY STATEMENT}

The raw data supporting the conclusions of this article will be made available by the authors, upon reasonable request. 


\section{ETHICS STATEMENT}

The animal study was reviewed and approved by Institutional Animal Care and Use Committee at Jinan University or Case Western Reserve University.

\section{AUTHOR CONTRIBUTIONS}

JT, XY, and YZ conceived the study, designed the experiments and wrote the manuscript with the help of co-authors. FW conducted all the majority of experiments and facilitated data analysis. FP and XG carried out the xenograft tumor study. All authors contributed to the article and approved the submitted version.

\section{REFERENCES}

Abbas, T., and Dutta, A. (2009). p21 in cancer: intricate networks and multiple activities. Nat. Rev. Cancer 9 (6), 400-414. doi: 10.1038/nrc2657

Andujar, I., Rios, J. L., Giner, R. M., and Recio, M. C. (2013). Pharmacological properties of shikonin - a review of literature since 2002. Planta Med. 79 (18), 1685-1697. doi: 10.1055/s-0033-1350934

Chang, I. C., Huang, Y. J., Chiang, T. I., Yeh, C. W., and Hsu, L. S. (2010). Shikonin induces apoptosis through reactive oxygen species/extracellular signalregulated kinase pathway in osteosarcoma cells. Biol. Pharm. Bull. 33 (5), 816-824. doi: 10.1248/bpb.33.816

Chen, X., Yang, L., Oppenheim, J. J., and Howard, O. M. Z. (2002). Cellular pharmacology studies of shikonin derivatives. Phytother. Res. 16 (3), 199-209. doi: $10.1002 /$ ptr.1100

Chen, X., Yang, L., Zhang, N., Turpin, J. A., Buckheit, R. W., Osterling, C., et al. (2003). Shikonin, a component of chinese herbal medicine, inhibits chemokine receptor function and suppresses human immunodeficiency virus type 1 . Antimicrob. Agents Chemother. 47 (9), 2810-2816. doi: 10.1128/ AAC.47.9.2810-2816.2003

Chen, J., Xie, J., Jiang, Z., Wang, B., Wang, Y., and Hu, X. (2011). Shikonin and its analogs inhibit cancer cell glycolysis by targeting tumor pyruvate kinase-M2. Oncogene 30 (42), 4297-4306. doi: 10.1038/onc.2011.137

Chen, Y., Wang, T., Du, J., Li, Y., Wang, X., Zhou, Y., et al. (2018). The Critical Role of PTEN/PI3K/AKT Signaling Pathway in Shikonin-Induced Apoptosis and Proliferation Inhibition of Chronic Myeloid Leukemia. Cell Physiol. Biochem. 47 (3), 981-993. doi: 10.1159/000490142

Duriez, P. J., and Shah, G. M. (1997). Cleavage of poly(ADP-ribose) polymerase: a sensitive parameter to study cell death. Biochem. Cell Biol. 75 (4), 337-349. doi: 10.1139/097-043

Fernandes-Alnemri, T., Litwack, G., and Alnemri, E. S. (1994). CPP32, a novel human apoptotic protein with homology to Caenorhabditis elegans cell death protein Ced-3 and mammalian interleukin-1 beta-converting enzyme. J. Biol. Chem. 269 (49), 30761-30764.

Funderburk, S. F., Wang, Q. J., and Yue, Z. (2010). The Beclin 1-VPS34 complexat the crossroads of autophagy and beyond. Trends Cell Biol. 20 (6), 355-362. doi: 10.1016/j.tcb.2010.03.002

Han, H. W., Zheng, C. S., Chu, S. J., Sun, W. X., Han, L. J., Yang, R. W., et al. (2018). The evaluation of potent antitumor activities of shikonin coumarincarboxylic acid, PMMB232 through HIF-1 alpha-mediated apoptosis. Biomed. Pharmacother. 97, 656-666. doi: 10.1016/j.biopha.2017.10.159

Han, X., Kang, K. A., Piao, M. J., Zhen, A. X., Hyun, Y. J., Kim, H. M., et al. (2019). Shikonin Exerts Cytotoxic Effects in Human Colon Cancers by Inducing Apoptotic Cell Death via the Endoplasmic Reticulum and MitochondriaMediated Pathways. Biomol. Ther. (Seoul). 27 (1), 41-47. doi: 10.4062/ biomolther.2018.047

Hoffman, W. H., Biade, S., Zilfou, J. T., Chen, J., and Murphy, M. (2002). Transcriptional repression of the anti-apoptotic survivin gene by wild type p53. J. Biol. Chem. 277 (5), 3247-3257. doi: 10.1074/jbc.M106643200

Jang, S. Y., Hong, D., Jeong, S. Y., and Kim, J. H. (2015). Shikonin causes apoptosis by up-regulating p73 and down-regulating ICBP90 in human cancer cells.

\section{FUNDING}

JT is supported by National Science Foundation of China (Nos. $81728022,81673320)$ and $\mathrm{YZ}$ is supported in part (30\%) by NCI (CA230453).

\section{SUPPLEMENTARY MATERIAL}

The Supplementary Material for this article can be found online at: https://www.frontiersin.org/articles/10.3389/fphar.2020.00861/ full\#supplementary-material

Biochem. Biophys. Res. Commun. 465 (1), 71-76. doi: 10.1016/ j.bbrc.2015.07.131

Kamada, Y., Funakoshi, T., Shintani, T., Nagano, K., Ohsumi, M., and Ohsumi, Y. (2000). Tor-mediated induction of autophagy via an Apg1 protein kinase complex. J. Cell Biol. 150 (6), 1507-1513. doi: 10.1083/jcb.150.6.1507

Karimian, A., Ahmadi, Y., and Yousefi, B. (2016). Multiple functions of p21 in cell cycle, apoptosis and transcriptional regulation after DNA damage. DNA Repair (Amst) 42, 63-71. doi: 10.1016/j.dnarep.2016.04.008

Kastenhuber, E. R., and Lowe, S. W. (2017). Putting p53 in Context. Cell 170 (6), 1062-1078. doi: 10.1016/j.cell.2017.08.028

Kim, H. J., Hwang, K. E., Park, D. S., Oh, S. H., Jun, H. Y., Yoon, K. H., et al. (2017). Shikonin-induced necroptosis is enhanced by the inhibition of autophagy in non-small cell lung cancer cells. J. Transl. Med. 15 (1), 123. doi: 10.1186/s12967-017-1223-7

Kundu, M., and Thompson, C. B. (2008). Autophagy: basic principles and relevance to disease. Annu. Rev. Pathol. 3, 427-455. doi: 10.1146/ annurev.pathmechdis.2.010506.091842

Levine, B., and Kroemer, G. (2008). Autophagy in the pathogenesis of disease. Cell 132 (1), 27-42. doi: 10.1016/j.cell.2007.12.018

Li, M. Y., Mi, C., Wang, K. S., Wang, Z., Zuo, H. X., Piao, L. X., et al. (2017). Shikonin suppresses proliferation and induces cell cycle arrest through the inhibition of hypoxia-inducible factor-1alpha signaling. Chem. Biol. Interact. 274, 58-67. doi: 10.1016/j.cbi.2017.06.029

Lin, K. H., Huang, M. Y., Cheng, W. C., Wang, S. C., Fang, S. H., Tu, H. P., et al. (2018). RNA-seq transcriptome analysis of breast cancer cell lines under shikonin treatment. Sci. Rep. 8 (1), 2672. doi: 10.1038/s41598-018-21065-X

Lu, B., Wang, Z., Ding, Y., Wang, X., Lu, S., Wang, C., et al. (2018). RIP1 and RIP3 contribute to shikonin-induced glycolysis suppression in glioma cells via increase of intracellular hydrogen peroxide. Cancer Lett. 425, 31-42. doi: 10.1016/j.canlet.2018.03.046

Mani, H., Sidhu, G. S., Singh, A. K., Gaddipati, J., Banaudha, K. K., Raj, K., et al. (2004). Enhancement of wound healing by shikonin analogue 93/637 in normal and impaired healing. Skin Pharmacol. Physiol. 17 (1), 49-56. doi: 10.1159/000074063

Mao, X., Yu, C. R., Li, W. H., and Li, W. X. (2008). Induction of apoptosis by shikonin through a ROS/JNK-mediated process in Bcr/Abl-positive chronic myelogenous leukemia (CML) cells. Cell Res. 18 (8), 879-888. doi: 10.1038/cr.2008.86

Matsuura, A., Tsukada, M., Wada, Y., and Ohsumi, Y. (1997). Apg1p, a novel protein kinase required for the autophagic process in Saccharomyces cerevisiae. Gene 192 (2), 245-250. doi: 10.1016/S0378-1119(97)00084-X

Mizushima, N., and Komatsu, M. (2011). Autophagy: renovation of cells and tissues. Cell 147 (4), 728-741. doi: 10.1016/j.cell.2011.10.026

Ni, F., Huang, X., Chen, Z., Qian, W., and Tong, X. (2018). Shikonin exerts antitumor activity in Burkitt's lymphoma by inhibiting C-MYC and PI3K/ AKT/mTOR pathway and acts synergistically with doxorubicin. Sci. Rep. 8 (1), 3317. doi: 10.1038/s41598-018-21570-z

Nicholson, D. W., Ali, A., Thornberry, N. A., Vaillancourt, J. P., Ding, C. K., Gallant, M., et al. (1995). Identification and inhibition of the ICE/CED-3 protease necessary for mammalian apoptosis. Nature 376 (6535), 37-43. doi: $10.1038 / 376037 \mathrm{a} 0$ 
Nigorikawa, K., Yoshikawa, K., Sasaki, T., Iida, E., Tsukamoto, M., Murakami, H., et al. (2006). A naphthoquinone derivative, shikonin, has insulin-like actions by inhibiting both phosphatase and tensin homolog deleted on chromosome 10 and tyrosine phosphatases. Mol. Pharmacol. 70 (3), 1143-1149. doi: 10.1124/mol.106.025809

Papageorgiou, V. P., Assimopoulou, A. N., Couladouros, E. A., Hepworth, D., and Nicolaou, K. C. (1999). The chemistry and biology of alkannin, shikonin, and related naphthazarin natural products. Angewandte Chem. Intern. Ed. 38 (3), 270 301. doi: 10.1002/(SICI)1521-3773(19990201)38:3<270::AID-ANIE270>3.0.CO;2-0

Peti, W., and Page, R. (2013). Molecular basis of MAP kinase regulation. Protein Sci. 22 (12), 1698-1710. doi: 10.1002/pro.2374

Qiu, H. Y., Fu, J. Y., Yang, M. K., Han, H. W., Wang, P. F., Zhang, Y. H., et al. (2017). Identification of new shikonin derivatives as STAT3 inhibitors. Biochem. Pharmacol. 146, 74-86. doi: 10.1016/j.bcp.2017.10.009

Sankawa, U., Ebizuka, Y., Miyazaki, T., Isomura, Y., and Otsuka, H. (1977). Antitumor activity of shikonin and its derivatives. Chem. Pharm. Bull. (Tokyo) 25 (9), 2392-2395. doi: 10.1248/cpb.25.2392

Shan, Z. L., Zhong, L., Xiao, C. L., Gan, L. G., Xu, T., Song, H., et al. (2017). Shikonin suppresses proliferation and induces apoptosis in human leukemia NB4 cells through modulation of MAPKs and cMyc. Mol. Med. Rep. 16 (3), 3055-3060. doi: 10.3892/mmr.2017.6965

Shen, X. J., Wang, H. B., Ma, X. Q., and Chen, J. H. (2012). beta,betaDimethylacrylshikonin induces mitochondria dependent apoptosis through ERK pathway in human gastric cancer SGC-7901 cells. PloS One 7 (7), e41773. doi: 10.1371/journal.pone.0041773

Tanaka, S., Tajima, M., Tsukada, M., and Tabata, M. (1986). A comparative study on anti-inflammatory activities of the enantiomers, shikonin and alkannin. J. Nat. Prod. 49 (3), 466-469. doi: 10.1021/np50045a014

Tang, J. C., Ren, Y. G., Zhao, J., Long, F., Chen, J. Y., and Jiang, Z. (2018a). Shikonin enhances sensitization of gefitinib against wild-type EGFR non-small cell lung cancer via inhibition PKM2/stat3/cyclinD1 signal pathway. Life Sci. 204, 71-77. doi: 10.1016/j.lfs.2018.05.012

Tang, J. C., Zhao, J., Long, F., Chen, J. Y., Mu, B., Jiang, Z., et al. (2018b). Efficacy of Shikonin against Esophageal Cancer Cells and its possible mechanisms in vitro and in vivo. J. Cancer 9 (1), 32-40. doi: 10.7150/jca.21224

Wang, F., Yao, X., Zhang, Y., and Tang, J. (2019). Synthesis, biological function and evaluation of Shikonin in cancer therapy. Fitoterapia 134, 329-339. doi: 10.1016/j.fitote.2019.03.005

Wirth, M., Joachim, J., and Tooze, S. A. (2013). Autophagosome formation-the role of ULK1 and Beclin1-PI3KC3 complexes in setting the stage. Semin. Cancer Biol. 23 (5), 301-309. doi: 10.1016/j.semcancer.2013.05.007

Wu, Z., Wu, L. J., Tashiro, S., Onodera, S., and Ikejima, T. (2005). Phosphorylated extracellular signal-regulated kinase up-regulated p53 expression in shikonininduced HeLa cell apoptosis. Chin. Med. J. (Engl.) 118 (8), 671-677.

Xiong, Y., Hannon, G. J., Zhang, H., Casso, D., Kobayashi, R., and Beach, D. (1993). p21 is a universal inhibitor of cyclin kinases. Nature 366 (6456), 701704. doi: $10.1038 / 366701 \mathrm{a} 0$
Zhai, T., Hei, Z., Ma, Q., Liang, H., Xu, Y., Zhang, Y., et al. (2017). Shikonin induces apoptosis and G0/G1 phase arrest of gallbladder cancer cells via the JNK signaling pathway. Oncol. Rep. 38 (6), 3473-3480. doi: 10.3892/ or.2017.6038

Zhang, F. L., Wang, P., Liu, Y. H., Liu, L. B., Liu, X. B., Li, Z., et al. (2013). Topoisomerase I inhibitors, shikonin and topotecan, inhibit growth and induce apoptosis of glioma cells and glioma stem cells. PloS One 8 (11), e81815. doi: 10.1371/journal.pone.0081815

Zhang, F. Y., Hu, Y., Que, Z. Y., Wang, P., Liu, Y. H., Wang, Z. H., et al. (2015). Shikonin Inhibits the Migration and Invasion of Human Glioblastoma Cells by Targeting Phosphorylated beta-Catenin and Phosphorylated PI3K/Akt: A Potential Mechanism for the Anti-Glioma Efficacy of a Traditional Chinese Herbal Medicine. Int. J. Mol. Sci. 16 (10), 23823-23848. doi: 10.3390/ ijms 161023823

Zhang, Y., Sun, B., Huang, Z., Zhao, D. W., and Zeng, Q. (2018). Shikonin Inhibites Migration and Invasion of Thyroid Cancer Cells by Downregulating DNMT1. Med. Sci. Monit. 24, 661-670. doi: 10.12659/MSM.908381

Zhao, Q., Assimopoulou, A. N., Klauck, S. M., Damianakos, H., Chinou, I., Kretschmer, N., et al. (2015). Inhibition of c-MYC with involvement of ERK/JNK/MAPK and AKT pathways as a novel mechanism for shikonin and its derivatives in killing leukemia cells. Oncotarget 6 (36), 38934-38951. doi: 10.18632 /oncotarget.5380

Zheng, H., Huang, Q., Huang, S., Yang, X., Zhu, T., Wang, W., et al. (2018). Senescence Inducer Shikonin ROS-Dependently Suppressed Lung Cancer Progression. Front. Pharmacol. 9, 519. doi: 10.3389/fphar.2018.00519

Zhou, G., Yang, Z., Wang, X., Tao, R., and Zhou, Y. (2017). TRAIL Enhances Shikonin Induced Apoptosis through ROS/JNK Signaling in Cholangiocarcinoma Cells. Cell Physiol. Biochem. 42 (3), 1073-1086. doi: $10.1159 / 000478758$

Zhu, J., Zhao, L., Luo, B., and Sheng, W. (2019). Shikonin regulates invasion and autophagy of cultured colon cancer cells by inhibiting yes-associated protein. Oncol. Lett. 18 (6), 6117-6125. doi: 10.3892/ol.2019.10980

Zhuang, S., and Schnellmann, R. G. (2006). A death-promoting role for extracellular signal-regulated kinase. J. Pharmacol. Exp. Ther. 319 (3), $991-$ 997. doi: 10.1124/jpet.106.107367

Conflict of Interest: The authors declare that the research was conducted in the absence of any commercial or financial relationships that could be construed as a potential conflict of interest.

Copyright (c) 2020 Wang, Mayca Pozo, Tian, Geng, Yao, Zhang and Tang. This is an open-access article distributed under the terms of the Creative Commons Attribution License (CC BY). The use, distribution or reproduction in other forums is permitted, provided the original author(s) and the copyright owner(s) are credited and that the original publication in this journal is cited, in accordance with accepted academic practice. No use, distribution or reproduction is permitted which does not comply with these terms. 